\title{
Über die «Verbesserung» des Menschen mit pharmakologischen Wirkstoffen*
}

Nationale Ethikkommission im Bereich der Humanmedizin NEK-CNE

\footnotetext{
* Bei diesem Artikel handelt es sich um eine Kurzversion. Literaturverweise befinden sich in der vollständigen Fassung der Stellungnahme, die unter www.saez.ch $\rightarrow$ Aktuelle Nummer oder $\rightarrow$ Archiv $\rightarrow 2011 \rightarrow 43$ sowie unter www.nek-cne.ch verfügbar ist.
}

\section{Korrespondenz:}

Susanne Brauer, PhD

Geschäftsstelle

Nationale Ethikkommission im Bereich der Humanmedizin NEK-CNE

c/o Bundesamt für Gesundheit

Seilerstrasse 8

CH-3003 Bern

Tel. 0313249365

susanne.brauer(at)bag.admin.ch
Es liegt in der Natur des Menschen, dass er sowohl seine körperlichen und geistigen Kapazitäten steigern als auch seine emotionalen und sozialen Fähigkeiten verbessern will. Manche Ethik hält ein solches Streben sogar für eine moralische Pflicht. Die Nationale Ethikkommission im Bereich der Humanmedizin (NEKCNE) ist dagegen der Auffassung, dass es auf die Mittel zur «Verbesserung» und auch auf die gesundheitlichen und sozialen Folgen eines Konsums von vermeintlich leistungssteigernden Wirkstoffen ankommt. Nicht alle Mittel und Folgen sind vom ethischen Standpunkt aus unproblematisch. Am Beispiel pharmakologischer Wirkstoffe, die bereits sowohl im Alltag als auch im Beruf und in Bildungseinrichtungen zur Leistungssteigerung konsumiert werden, legt die NEK-CNE ihre ethischen Überlegungen bewusst in thesenhafter Form dar und leitet daraus Empfehlungen ab. Ziel ist, die Frage nach möglichen gesundheitlichen und gesellschaftlichen Risiken einer nicht-therapeutischen «Verbesserung» des Menschen aufzuwerfen. Dies geschieht mit der Absicht, zum einen vertiefte und langfristige ethische, medizinische, sozialwissenschaftliche, psychologische und suchtpräventive Forschungsvorhaben anzustossen, zum anderen Politik und Öffentlichkeit zur erhöhten Aufmerksamkeit anzuregen.

\section{Enhancement - eine Sache der persönlichen Freiheit?}

Ein wesentlicher Ausgangspunkt der ethischen Überlegungen der NEK-CNE ist, Enhancement nicht für eine «reine Privatsache» zu halten. Damit weicht sie von der Ansicht ab, Enhancement dann für ethisch unbedenklich zu erachten, wenn sich mündige Erwachsene freiwillig zur Einnahme von Pharmaka ohne medizinische Indikation entscheiden sowie über deren Wirkungen und gesundheitsschädliche Nebenwirkungen nicht getäuscht werden. Nach dieser Ansicht muss weder eine explizite Aufklärung über mögliche Nebenwirkungen stattfinden, z.B. durch einen Beipackzettel, noch die Nicht-Schädlichkeit des Produktes gewährleistet sein. Analog zum erhöhten Alkoholgenuss stehe es dem einzelnen Menschen frei, sich selbst zu schaden und sich bei Bedarf über die Wirkungsweisen eines Stoffes in Eigeninitiative und Eigenverantwortung kundig zu machen. Diese Freiheit sogar zum selbstschädigenden Verhalten wird auch von der NEK-CNE nicht grundsätzlich abgelehnt. Trotzdem greift für die NEKCNE die dargestellte Position im Fall von Enhancement zu kurz.

\section{Menschenbild und Medizin im Wandel}

Zwei Argumente sprechen gegen die skizzierte Ansicht: Erstens bezweifelt die Kommission, dass das Bild eines rationalen Individuums, das frei von sozialen Einflüssen, Erwartungen und Beziehungen entscheidet und dann gemäss seinem Wissen und seinen Interessen handelt, realistisch ist. Zweitens blendet die Position, da sie sich allein auf das Handeln des einzelnen Individuums konzentriert, das gesellschaftliche Umfeld aus, in dem Enhancement erwartet wird oder schon stattfindet. Die gesellschaftlichen Folgen sind jedoch Aspekte, die nach Ansicht der NEK-CNE in die ethische Bewertung von Enhancement mit einfliessen müssen. Wenn sich die Erwartungen über die menschliche Leistungsfähigkeit ändern und erwartet wird, dass der Mensch pharmakologische Mittel zur Leistungssteigerung einnimmt, ändert sich damit in einer Gesellschaft auch das Menschenbild.

Zudem hat diese Entwicklung tiefgreifende Auswirkungen auf das Berufsbild der Ärzteschaft, denn es werden leistungssteigernde Substanzen nachgefragt, die verschreibungspflichtig sind. Wenn die Verschreibung jedoch nicht mehr therapeutischen Massstäben folgt oder die Indikationen ständig ausgeweitet werden, dann entwickelt sich die ärztliche Tätigkeit in Richtung einer wunscherfüllenden Medizin, und das ärztliche Ethos erfährt einen bedenklichen Wandel. Die Ärzteschaft sollte sich bewusst sein, dass sie infolge ihrer Arbeit, ihrer Autorität und ihres Ansehens in der Gesellschaft deren Werte und Massstäbe beeinflusst. Zudem wird durch Enhancement der Wettbewerb in der Gesellschaft mit Mitteln fortgesetzt, die ursprünglich der kurativen Medizin vorbehalten waren.

\section{Gesundheitsrisiken und \\ Persönlichkeitsentwicklung}

Dass die Einnahme von pharmakologischen Mitteln das persönliche Wohlbefinden steigern kann, wie einige Erfahrungsberichte bestätigen, könnte als Gewinn gesehen werden. Allerdings ist die tatsächliche Steigerung der eigenen Leistungsfähigkeit nur vorübergehend und wird oft überschätzt. Zudem kann nach heutigem Stand eine kognitive Fähigkeit (z.B. Kapazitäten des Kurzzeitgedächnisses) nur auf Kosten anderer kognitiver oder emotionaler Fähigkeiten (z. B. assoziatives Denkvermögen, Tiefgründigkeit, Frustrationstoleranz) maximiert werden. Es ist häufig nicht auszuschliessen, dass ein Konsum ebenfalls zu unerwünschten Nebenwirkungen oder unter 
Umständen sogar zur psychischen oder physischen Abhängigkeit, also Sucht führen kann. Andere Formen, seine Persönlichkeit zu entwickeln und seine Lebensqualität zu verbessern, gehen aus dem Blickfeld verloren. Training, Selbsterziehung, Meditation usw. sind zwar zeitaufwendig, gleichwohl effektiv und beim richtigen Mass sogar gesundheitsförderlich.

\section{Gesellschaftliche Risiken}

Ein verbreiteter Konsum schafft nicht nur persönliche Gesundheitsrisiken, sondern verändert auch die Erwartungen der Gesellschaft an das zu erbringende Leistungsniveau. Zum Beispiel nehmen die Belastungen am Arbeitsplatz zu und damit steigt die Gefahr psychischer Erkrankungen. Wenn erhöhte Anforderungen medikamentös bewältigt werden, anstatt gegebenenfalls Arbeitsstrukturen und -bedingungen anzupassen, entspräche dies einer Logik von gesellschaftlich anerkanntem Enhancement und würde diejenigen unter Druck setzen, die sich dem Konsum leistungssteigernder Produkte verweigern. Die Freiwilligkeit von Enhancement wäre damit eingeschränkt. Dass diese Befürchtungen berechtigt sind, zeigen Umfragen: Nicht um Spitzenleistungen zu erzielen, sondern um «mithalten zu können» und den «Stress zu bewältigen», wird Enhancement betrieben. Benachteiligt werden auch diejenigen, bei denen die Wirkstoffe wenig oder keinen Effekt zeigen.

\section{Plädoyer für menschliche Vielfalt}

Wenn Enhancement grosse Verbreitung fände, würde eine Vielfalt von Lebensformen und Begabungsunterschieden eingeschränkt werden. Ein Verlust an Vielfalt wäre jedoch nicht wünschenswert, weil anderenfalls die unterschiedlichen Eigenarten und Besonderheiten der Menschen eingeebnet werden und die Toleranz gegenüber Andersartigkeit abnehmen würde. Wenn der Uniformitätsdruck in einem Mass steigt, dass eine andersartige Begabung oder auch eine Behinderung als «Eigenverschulden» angesehen werden, weil man doch dagegen ein Mittel einnehmen sollte, dann ginge dies auch mit einem Verlust an Gerechtigkeit einher.

\section{Kollektive Prävention und Gesundheitspolitik}

Die Antwort auf die Frage, welche gesundheitspolitischen Strategien legitim sind oder aber in die Persönlichkeitsrechte eingreifen, hängt von kulturellen und historischen Prägungen ab. So wäre es durchaus denkbar, dass unter der Flagge «Prävention und Gesundheitsförderung» (vormalige) Enhancement-Methoden in die Gesundheitspolitik aufgenommen würden. Es darf aber nicht nur eine Frage der Sprachregelung sein, ob beispielsweise diagnostische Methoden (z.B. bei der Schwangerschaftsvorsorge) oder die Anreicherung von Grundnahrungsmitteln mit Zusatzstoffen (z.B. Jod) eine Form von Enhancement oder eine Massnahme im Sinne einer kollektiven Prävention ist.
Dieser Unterschied ist jedoch grundlegend. Während präventive Massnahmen Krankheiten verhindern sollen und daher staatlich empfohlen und solidarisch finanziert werden, erfährt Enhancement nach Ansicht der NEK-CNE zu Recht keine derartige Unterstützung. Die NEK-CNE rät zur Wachsamkeit, dass ein mögliches Verschieben der Grenze zwischen den Bereichen Enhancement und Prävention medizinisch sinnvoll erfolge und aus der Sicht der Gesamtgesellschaft vertretbar sei und nicht etwa von kommerziellen Interessen angetrieben werde.

\section{Enhancement bei Kindern}

Besondere Aufmerksamkeit verdient das Enhancement bei Kindern. Hier ist eine steigende Tendenz zu beobachten, die nicht (voll) urteilsfähige Personen betreffen. Diese Tendenz wird durch die Motivation der Eltern, nur «das Beste» für ihr Kind zu wollen, unterstützt. Dabei wird oft «das Beste» nur mit Blick auf das zukünftige Leben in einer Wettbewerbsgesellschaft definiert.

Nach Angaben des US President's Council on Bioethics stimmt der Einsatz von Psychopharmaka wie Ritalin $^{\circledR}$ und Concerta ${ }^{\circledR}$ nicht mit der Anzahl der gestellten Diagnosen und Therapieindikationen überein, und es ist eine Tendenz hinsichtlich des Geschlechts (Knaben), des Alters (jüngere Kinder) und der Region (Stadt) festzustellen. Eine solche Tendenz ist neben sprachregionalen Unterschieden auch in der Schweiz zu beobachten. Leider liegt trotz zahlreicher parlamentarischer Vorstösse kein differenzierter, repräsentativer Bericht aktuelleren Datums zur gesamtschweizerischen Verschreibungspraxis und zum Verbrauch von Psychopharmaka bei Kindern vor. Ein solcher Bericht wäre jedoch aufschlussreich, um auch die Ursachen klären zu können, warum z. B. Methylphenidat, unter anderem bekannt als Ritalin ${ }^{\circledR}$, das seit über 55 Jahren in der Schweiz in Gebrauch ist, in den letzten 15 Jahren so viel häufiger zum Einsatz gekommen ist.

Aus ethischer Perspektive ist die Tatsache von Belang, dass die Diagnose beispielsweise eines Aufmerksamkeitsdefizitsyndroms, eines oppositionellen Trotzverhaltens oder einer Angststörung eine fachliche Herausforderung darstellt, weil die Abgrenzung zwischen normalen und krankhaften kindlichen Verhaltensweisen schwierig zu ziehen ist. Ebenfalls ist anzunehmen, dass durch den Anstieg des Verbrauchs von Psychopharmaka sich auch die Standards verschieben bzw. verschoben haben, welche Verhaltensweisen eines Kindes oder Jugendlichen sozial verträglich und «normal» sind - oder eben als krankhaft eingestuft werden. Da die Diagnosestellung auch von solchen gesellschaftlichen Bewertungen sowie einem Interesse, dass sich Kinder im Kindergarten und in der Schule angepasst verhalten, beeinflusst ist, ist eine weitere Zunahme der Verschreibungen zu erwarten. Dieses Beispiel zeigt, dass die Abgrenzung zwischen Enhancement und Therapiebedürftigkeit kul- 
turell und historisch variabel ist - und damit auch ethischer Reflexion bedarf.

Selbstverständlich kann man gegenüber dieser Entwicklung positiv eingestellt sein, weil offenkundig wünschenswerte Eigenschaften des Kindes und damit seine soziale Integration gefördert werden. Solche Förderung wird von einigen sogar zur moralischen Pflicht erhoben. Die NEK-CNE hat hier jedoch Bedenken. Denn durch die Einnahme von pharmakologischen Wirkstoffen zu Zwecken des Enhancement wird das Verhalten des Kindes ohne jegliche Eigenleistung verändert. Darin liegt ein Eingriff in die Freiheit und die Persönlichkeitsrechte des Kindes. Weil pharmakologische Wirkstoffe zwar Verhaltensveränderungen verursachen, das Kind aber damit nicht lernt, wie es solche Verhaltensänderungen selbst erzielen kann, wird dem Kind eine wichtige Lernerfahrung für eigenverantwortliches Handeln vorenthalten: nämlich sein Verhalten durch eigene Entscheidungen - und nicht (allein) durch fremde Mittel - zu beeinflussen und damit Verantwortung übernehmen zu können. In diesem Sinne wird durch Enhancement die Freiheit des Kindes empfindlich eingeschränkt und es in seiner Persönlichkeitsentwicklung gehemmt.
Der Konsum pharmakologischer Mittel kann noch weitere Auswirkungen auf den Charakter haben, weil dem Kind vermittelt wird, dass es nur mit Hilfe solcher Mittel in sozial anerkannter Weise «funktioniert». Insofern seine Charaktereigenschaften medikamentös angepasst und von Psychopharmaka abhängig gemacht werden, hat es Folgen für seine Persönlichkeitsbildung und sein Selbstwertgefühl und könnte die Ausbildung von Mustern für Suchtverhalten begünstigen. Der Konformitätsdruck, unter dem Kinder vonseiten der Eltern und Bildungseinrichtungen stehen, erzwingt einen Standard an Normalität, der die Toleranz gegenüber Kindlichkeit abnehmen lässt. Auch könnte sich die Vielfalt von Temperamenten und Lebensweisen reduzieren und damit letztlich das Recht des Kindes auf einen offenen Lebensweg gefährdet werden. Die NEK-CNE plädiert dafür, die Lebensverhältnisse den Interessen und Bedürfnissen der Kinder anzupassen. Denn die Qualitäten der Kindheit, die nicht Aspekte des gesellschaftlichen Wettbewerbs und der Leistungsfähigkeit betreffen, sondern das Spielen, die Freundschaft und die erfolgsentlastete Musse ausmachen, könnten anderenfalls an Wertschätzung verlieren und damit auch die Kindheit selbst.

\section{Empfehlungen der NEK-CNE}

- Die Ärzteschaft sollte sich bewusst sein, dass sie mit einer breiten Verschreibungspraxis von Medikamenten für nicht-therapeutische Zwecke das Bild der Medizin in der Öffentlichkeit verändert, darüber hinaus das Menschenbild in der Gesellschaft beeinflusst.

- Die persönliche Freiheit ist grundsätzlich zu respektieren, sogar bei selbstschädigendem Verhalten, jedoch nicht auf Kosten gesellschaftlicher Grundwerte wie Gerechtigkeit und Toleranz gegenüber Andersartigkeit. Ausserdem sollten Verhaltensweisen mit Gesundheitsrisiken nicht ärztlich gefördert werden.

- Da auch frei zugängliche pharmakologische Wirkstoffe Gesundheitsrisiken bergen, sollten sie - wie auch die Gründe und Weisen ihrer Nutzung - eingehend erforscht und einer entsprechenden Kontrolle unterworfen werden.

- Bildungseinrichtungen sollten sich ihrer Verantwortung für die psychische Gesundheit ihrer Schutzbefohlenen und Arbeitgeber ihrer Pflichten gegenüber ihren Mitarbeitenden bewusst sein und einem zu hohen Leistungsdruck entgegenwirken.

- In der Gesundheitspolitik sollte Enhancement mit Prävention, also der Verhinderung von Krankheiten, weder verwechselt noch vermischt werden.

- Vor allem bei Kindern kann pharmakologisches Enhancement die Freiheit, die Persönlichkeitsrechte und die Persönlichkeitsentwicklung einschränken. Eltern, Bildungseinrichtungen und andere Sorgeberechtigte tragen hier eine besondere Verantwortung, sowohl für das einzelne Kind als auch für die zukünftigen Werte und Massstäbe unserer Gesellschaft.

- Die gegenwärtige Verschreibungspraxis von Psychopharmaka bei Kindern ist zu überprüfen, die Ursachen des höheren Verbrauchs sind zu klären und die Kinder vor übermässigem Gebrauch zu schützen. 


\section{Über die „Verbesserung“ des Menschen mit pharmakologischen Wirkstoffen}

\section{Nationale Ethikkommission im Bereich der Humanmedizin NEK-CNE}

Es liegt in der Natur des Menschen, dass er sowohl seine körperlichen und geistigen Kapazitäten steigern als auch seine emotionalen und sozialen Fähigkeiten verbessern will. Manche Ethik hält ein solches Streben sogar für eine moralische Pflicht [1,2]. Die Nationale Ethikkommission im Bereich der Humanmedizin (NEK-CNE) ist dagegen der Auffassung, dass es auf die Mittel zur "Verbesserung“ und auch auf die gesundheitlichen und sozialen Folgen eines Konsums von vermeintlich leistungssteigernden Wirkstoffen ankommt. Nicht alle Mittel und Folgen sind vom ethischen Standpunkt aus unproblematisch $[3,4,5,6,7,8,9,10$, 11, 12, 13]. Am Beispiel pharmakologischer Wirkstoffe, welche bereits sowohl im Altag als auch im Beruf und in Bildungseinrichtungen zur Leistungssteigerung konsumiert werden, legt die NEK-CNE ihre ethischen Überlegungen bewusst in thesenhafter Form dar und leitet daraus Empfehlungen ab. Ziel ist, die Frage nach möglichen gesundheitlichen und gesellschaftlichen Risiken einer nicht-therapeutischen „Verbesserung” des Menschen aufzuwerfen [14]. Dies geschieht mit der Absicht, zum einen vertiefte und langfristige ethische, medizinische, sozialwissenschaftliche, psychologische und suchtpräventive Forschungsvorhaben anzustossen, zum anderen Politik und Öffentlichkeit zur erhöhten Aufmerksamkeit anzuregen.

Es hat sich im Sprachgebrauch eingebürgert, für Veränderungen des Menschen, die nicht therapeutisch angezeigt sind, aber dennoch als eine „Verbesserung” menschlicher Fähigkeiten, Leistungen und Befindlichkeiten eingeschätzt werden, den englischen Ausdruck Enhancement zu übernehmen. ${ }^{1}$ Enhancement kann dabei nicht nur eine Leistungssteigerung oder Verhaltensänderung sein, welche die betroffene Person als einen Gewinn an Lebensqualität empfindet, sondern auch eine, die auch von ihrem Umfeld, zum Beispiel in der Schule und am Arbeitsplatz, eventuell sogar in der Familie und Partnerschaft, erwünscht ist. Ein vermehrter Konsum von pharmakologischen Wirkstoffen ist unter diesen Bedingungen zu erwarten, obwohl zur Zeit (noch) eine allgemeine Zurückhaltung der Schweizer Bevölkerung gegenüber Enhancement zu beobachten ist [15]. Dies könnte sich mit steigendem Verbrauch und damit einhergehend der vermehrten Anwendung solcher

\footnotetext{
${ }^{1}$ In der jüngsten Studie des Schweizer Zentrums für Technologiefolgen-Abschätzung (TA-Swiss) zum Thema „Human Enhancement“ wird Enhancement definiert als „alle medizinischen und biotechnologischen Interventionen, die darauf zielen, Menschen in ihren Fähigkeiten und ihrer Gestalt in einer Weise zu verändern, die in den jeweiligen soziokulturellen Kontexten als Verbesserung wahrgenommen wird, deren Zielsetzung nicht primär therapeutischer oder präventiver Art ist." [16, S. 5f.]
} 
Wirkstoffe, aber auch im Fall der Entdeckung neuer, nebenwirkungsärmerer oder -freier Substanzen ändern.

\section{Enhancement - eine Sache der persönlichen Freiheit?}

Ein wesentlicher Ausgangspunkt der ethischen Überlegungen der NEK-CNE ist, Enhancement nicht für eine „reine Privatsache“ zu halten. Damit weicht sie von der Ansicht ab, Enhancement dann für ethisch unbedenklich zu erachten, wenn sich mündige Erwachsene freiwillig zur Einnahme von Pharmaka ohne medizinische Indikation entscheiden, sowie über deren Wirkungen und gesundheitsschädlichen Nebenwirkungen nicht getäuscht werden [16]. Nach dieser Ansicht muss weder eine explizite Aufklärung über mögliche Nebenwirkungen stattfinden, z.B. durch einen Beipackzettel, noch die NichtSchädlichkeit des Produktes gewährleistet sein. Analog zum erhöhten Alkoholgenuss stehe es dem einzelnen Menschen frei, sich selbst zu schaden und sich bei Bedarf über die Wirkungsweisen eines Stoffes in Eigeninitiative und Eigenverantwortung kundig zu machen. Diese Freiheit sogar zum selbstschädigenden Verhalten wird auch von der NEK-CNE nicht grundsätzlich abgelehnt [17]. Trotzdem greift für die NEK-CNE die dargestellte Position im Fall von Enhancement zu kurz.

\section{Menschenbild und Medizin im Wandel}

Zwei Argumente sprechen gegen die skizzierte Ansicht: Erstens bezweifelt die Kommission, dass das Bild eines rationalen Individuums, das frei von sozialen Einflüssen, Erwartungen und Beziehungen entscheidet und dann gemäss seinem Wissen und seinen Interessen handelt, realistisch ist. Zweitens blendet die Position, da sie sich allein auf das Handeln des einzelnen Individuums konzentriert, das gesellschaftliche Umfeld aus, in dem Enhancement erwartet wird oder schon stattfindet. Die gesellschaftlichen Folgen sind jedoch Aspekte, die nach Ansicht der NEK-CNE in die ethische Bewertung von Enhancement mit einfliessen müssen. Wenn sich die Erwartungen über die menschliche Leistungsfähigkeit ändern und erwartet wird, dass der Mensch pharmakologische Mittel zur Leistungssteigerung einnimmt, ändert sich damit in einer Gesellschaft auch das Menschenbild.

Zudem hat diese Entwicklung tiefgreifende Auswirkungen auf das Berufsbild der Ärzteschaft, denn es werden leistungssteigernde Substanzen nachgefragt, die verschreibungspflichtig sind. Wenn die Verschreibung jedoch nicht mehr therapeutischen Massstäben folgt oder die Indikationen ständig ausgeweitet werden, dann entwickelt sich die ärztliche Tätigkeit in Richtung einer wunscherfüllenden Medizin, und das ärztliche Ethos erfährt einen bedenklichen Wandel [18]. Die Ärzteschaft sollte sich bewusst sein, dass sie in Folge ihrer Arbeit, ihrer Autorität und ihres Ansehens in der Gesellschaft deren Werte und Massstäbe beeinflusst. Zudem wird durch Enhancement der Wettbewerb in der 
Gesellschaft mit Mitteln fortgesetzt, die ursprünglich der kurativen Medizin vorbehalten waren.

\section{Gesundheitsrisiken und Persönlichkeitsentwicklung}

Dass die Einnahme von pharmakologischen Mitteln das persönliche Wohlbefinden steigern kann, wie einige Erfahrungsberichte bestätigen [19], könnte als Gewinn gesehen werden. Allerdings ist die tatsächliche Steigerung der eigenen Leistungsfähigkeit nur vorrübergehend und wird oft überschätzt [20, 21]. Zudem kann nach heutigem Stand eine kognitive Fähigkeit (z.B. Kapazitäten des Kurzzeitgedächnisses) nur auf Kosten anderer kognitiver oder emotionaler Fähigkeiten (z.B. assoziatives Denkvermögen, Tiefgründigkeit, Frustrationstoleranz) maximiert werden [20]. Es ist häufig nicht auszuschliessen, dass ein Konsum ebenfalls zu unerwünschten Nebenwirkungen oder unter Umständen sogar zur psychischen oder physischen Abhängigkeit, also Sucht führen kann [19]. Andere Formen, seine Persönlichkeit zu entwickeln und seine Lebensqualität zu verbessern, gehen aus dem Blickfeld verloren. Training, Selbsterziehung, Meditation usw. sind zwar zeitaufwendig, gleichwohl effektiv und beim richtigen Mass sogar gesundheitsförderlich.

\section{Gesellschaftliche Risiken}

Ein verbreiteter Konsum schafft nicht nur persönliche Gesundheitsrisiken, sondern verändert auch die Erwartungen der Gesellschaft an das zu erbringende Leistungsniveau. Zum Beispiel nehmen die Belastungen am Arbeitsplatz zu und damit steigt die Gefahr psychischer Erkrankungen [22]. Wenn erhöhte Anforderungen medikamentös bewältigt werden, anstatt gegebenenfalls Arbeitsstrukturen und -bedingungen anzupassen [23], entspräche dies einer Logik von gesellschaftlich anerkanntem Enhancement und würde diejenigen unter Druck setzen, die sich dem Konsum leistungssteigernder Produkte verweigern. Die Freiwilligkeit von Enhancement wäre damit eingeschränkt. Dass diese Befürchtungen berechtigt sind, zeigen Umfragen: Nicht um Spitzenleistungen zu erzielen, sondern um „mithalten zu können“ und den „Stress zu bewältigen“, wird Enhancement betrieben [16]. Benachteiligt werden auch diejenigen, bei denen die Wirkstoffe wenig oder keinen Effekt zeigen.

Derartigen Befürchtungen könnte man entgegenhalten, dass sich Enhancement auch einsetzen lässt, um natürliche Unterschiede in Begabung und Leistungsfähigkeit auszugleichen, mithin die Chancengleichheit in der Gesellschaft zu fördern. Deshalb sollte Enhancement vor allem den „Bedürftigen“ zugänglich gemacht werden. Die NEK-CNE überzeugt diese Argumentation aus folgenden Überlegungen nicht: 


\section{Plädoyer für menschliche Vielfalt}

Wenn Enhancement grosse Verbreitung fände, würde eine Vielfalt von Lebensformen und Begabungsunterschieden eingeschränkt werden. Ein Verlust an Vielfalt wäre jedoch nicht wünschenswert, weil anderenfalls die unterschiedlichen Eigenarten und Besonderheiten der Menschen eingeebnet werden und die Toleranz gegenüber Andersartigkeit abnehmen würde. Wenn der Uniformitätsdruck in einem Mass steigt, dass eine andersartige Begabung oder auch eine Behinderung als „Eigenverschulden“ angesehen werden, weil man doch dagegen ein Mittel einnehmen sollte, dann ginge dies auch mit einem Verlust an Gerechtigkeit einher.

\section{Kollektive Prävention und Gesundheitspolitik}

Die Antwort auf die Frage, welche gesundheitspolitischen Strategien legitim sind oder aber in die Persönlichkeitsrechte eingreifen, hängt von kulturellen und historischen Prägungen ab $[24,25]$. So wäre es durchaus denkbar, dass unter der Flagge „Prävention und Gesundheitsförderung" (vormalige) Enhancement-Methoden in die Gesundheitspolitik aufgenommen würden. Es darf aber nicht nur eine Frage der Sprachregelung sein, ob beispielsweise diagnostische Methoden (z.B. bei der Schwangerschaftsvorsorge) oder die Anreicherung von Grundnahrungsmitteln mit Zusatzstoffen (z.B. Jod) eine Form von Enhancement oder eine Massnahme im Sinne einer kollektiven Prävention sind. Dieser Unterschied ist jedoch grundlegend. Während präventive Massnahmen Krankheiten verhindern sollen und daher staatlich empfohlen und solidarisch finanziert werden, erfährt Enhancement nach Ansicht der NEK-CNE zu Recht keine derartige Unterstützung. Die NEKCNE rät zur Wachsamkeit, dass ein mögliches Verschieben der Grenze zwischen den Bereichen Enhancement und Prävention medizinisch sinnvoll erfolge und aus der Sicht der Gesamtgesellschaft vertretbar sei und nicht etwa von kommerziellen Interessen angetrieben werde.

\section{Enhancement bei Kindern}

Besondere Aufmerksamkeit verdient das Enhancement bei Kindern. Hier ist eine steigende Tendenz zu pharmakologischen Eingriffen zu beobachten, die noch nicht (voll) urteilsfähige Personen betreffen, über die Erwachsene, in der Regel die Eltern, auch in gesundheitlichen Belangen entscheiden dürfen. Diese Tendenz erfährt durch die Motivation der Eltern, nur "das Beste" für ihr Kind zu wollen und sicherzustellen, zusätzlich Auftrieb [26]. Dabei wird oft „das Beste" mit Blick auf das zukünftige Leben in der Gesellschaft definiert: Die Eltern wünschen in der Regel, dass das Kind im Wettbewerb um Ausbildung und Arbeitsplatz gut bestehe, indem vor allem seine kognitiven, aber auch emotionalen und sozialen Fähigkeiten verbessert und seine „Stressresistenz" gesteigert werden. Dieser Wettbewerb beginnt bereits sehr früh, verstärkt beim Schuleintritt. Bekanntlich zeigen Psychopharmaka auch bei 
gesunden Kindern Wirkung. Entsprechend gross ist der Anreiz für die Eltern, solche Mittel einzusetzen, um die Aufmerksamkeit und Konzentration des Kindes zu fördern und es damit konkurrenzfähiger zu machen. Eine derartige „Optimierung“ der kindlichen Fähigkeiten geschieht ohne Zeitaufwand und auch unbemerkt, so dass sich die Eltern kritischen Bemerkungen nicht stellen müssen.

Nach Angaben des US President's Council on Bioethics stimmt der Einsatz von Psychopharmaka wie Ritalin ${ }^{\circledR}$ und Concerta ${ }^{\circledR}$ nicht mit der Anzahl der gestellten Diagnosen und Therapieindikationen überein, und es ist eine Tendenz hinsichtlich des Geschlechts (Knaben), des Alters (jüngere Kinder) und der Region (Stadt) festzustellen [26, S. 75; 80]. Eine solche Tendenz ist neben sprachregionalen Unterschieden auch in der Schweiz zu beobachten [27, 28]. Der Verbrauch an Ritalin ${ }^{\circledR}$ in der Schweiz ist zudem zwischen 1996 und 2000 von $13,7 \mathrm{~kg}$ auf $69 \mathrm{~kg}$ markant gestiegen, vor allem in der Altersgruppe der 5- bis 14jährigen, [28]. Die durchschnittliche Dosierung wurde innerhalb dieser vier Jahre um ca. 10\% erhöht [28]. Leider liegt trotz zahlreicher parlamentarischer Vorstösse kein differenzierter, repräsentativer Bericht aktuelleren Datums zur gesamtschweizerischen Verschreibungspraxis und zum Verbrauch von Psychopharmaka bei Kindern vor [27, 28, 29, 30]. Ein solcher Bericht wäre jedoch aufschlussreich, um auch die Ursachen klären zu können, warum z.B. Methylphenidat, unter anderem bekannt als Ritalin ${ }^{\circledR}$, das seit über 55 Jahren in der Schweiz in Gebrauch ist, in den letzten 15 Jahren so viel häufiger zum Einsatz gekommen ist.

Aus ethischer Perspektive ist die Tatsache von Belang, dass die Diagnose beispielsweise eines Aufmerksamkeitsdefizitsyndroms, eines oppositionellen Trotzverhaltens oder einer Angststörung eine fachliche Herausforderung darstellt, weil die Abgrenzung zwischen normalen und krankhaften kindlichen Verhaltensweisen schwierig zu ziehen ist [31]. Ebenfalls ist anzunehmen, dass durch den Anstieg des Verbrauchs von Psychopharmaka sich auch die Standards verschieben bzw. verschoben haben, welche Verhaltensweisen eines Kindes oder Jugendlichen sozial verträglich und "normal“ sind - oder eben als krankhaft eingestuft werden. Da die Diagnosestellung auch von solchen gesellschaftlichen Bewertungen sowie einem Interesse, dass sich Kinder im Kindergarten und in der Schule angepasst verhalten, beeinflusst ist, ist eine weitere Zunahme der Verschreibungen zu erwarten. Dieses Beispiel zeigt, dass die Abgrenzung zwischen Enhancement und Therapiebedürftigkeit kulturell und historisch variabel ist - und damit auch ethischer Reflexion bedarf.

Selbstverständlich kann man gegenüber dieser Entwicklung positiv eingestellt sein, weil offenkundig wünschenswerte Eigenschaften des Kindes und damit seine soziale Integration 
gefördert werden. Solche Förderung wird von einigen sogar zur moralischen Pflicht erhoben. Die NEK-CNE hat hier jedoch Bedenken. Denn durch die Einnahme von pharmakologischen Wirkstoffen zu Zwecken des Enhancement wird das Verhalten des Kindes ohne jegliche Eigenleistung verändert [32]. Darin liegt ein Eingriff in die Freiheit und die Persönlichkeitsrechte des Kindes. Weil pharmakologische Wirkstoffe zwar Verhaltensveränderungen verursachen, das Kind aber damit nicht lernt, wie es solche Verhaltensänderungen selbst erzielen kann, wird dem Kind eine wichtige Lernerfahrung für eigenverantwortliches Handeln vorenthalten [26]: nämlich sein Verhalten durch eigene Entscheidungen - und nicht (allein) durch fremde Mittel - zu beeinflussen und damit Verantwortung übernehmen zu können. In diesem Sinne wird durch Enhancement die Freiheit des Kindes empfindlich eingeschränkt und es in seiner Persönlichkeitsentwicklung gehemmt.

Der Konsum pharmakologischer Mittel kann noch weitere Auswirkungen auf den Charakter haben, weil dem Kind vermittelt wird, dass es nur mit Hilfe solcher Mittel in sozial anerkannter Weise "funktioniert". Insofern seine Charaktereigenschaften medikamentös angepasst und von Psychopharmaka abhängig gemacht werden, hat es Folgen für seine Persönlichkeitsbildung und sein Selbstwertgefühl und könnte die Ausbildung von Mustern für Suchtverhalten begünstigen [19]. Der Konformitätsdruck, unter dem Kinder von Seiten der Eltern und Bildungseinrichtungen stehen, erzwingt einen Standard an Normalität, der die Toleranz gegenüber Kindlichkeit abnehmen lässt. Auch könnte sich die Vielfalt von Temperamenten und Lebensweisen reduzieren und damit letztlich das Recht des Kindes auf einen offenen Lebensweg gefährdet werden. Die NEK-CNE plädiert dafür, die Lebensverhältnisse den Interessen und Bedürfnissen der Kinder anzupassen. Denn die Qualitäten der Kindheit, die nicht Aspekte des gesellschaftlichen Wettbewerbs und der Leistungsfähigkeit betreffen, sondern das Spielen, die Freundschaft und die erfolgsentlastete Musse ausmachen, könnten anderenfalls an Wertschätzung verlieren - und damit auch die Kindheit selbst. 


\section{Empfehlungen der NEK-CNE}

1. Die Ärzteschaft sollte sich bewusst sein, dass sie mit einer breiten Verschreibungspraxis von Medikamenten für nicht-therapeutische Zwecke das Bild der Medizin in der Öffentlichkeit verändert, darüber hinaus das Menschenbild in der Gesellschaft beeinflusst.

2. Die persönliche Freiheit ist grundsätzlich zu respektieren, sogar bei selbstschädigendem Verhalten, jedoch nicht auf Kosten gesellschaftlicher Grundwerte wie Gerechtigkeit und Toleranz gegenüber Andersartigkeit. Ausserdem sollten Verhaltensweisen mit Gesundheitsrisiken nicht ärztlich gefördert werden.

3. Da auch frei zugängliche pharmakologische Wirkstoffe Gesundheitsrisiken bergen, sollten sie - wie auch die Gründe und Weisen ihrer Nutzung - eingehend erforscht und einer entsprechenden Kontrolle unterworfen werden.

4. Bildungseinrichtungen sollten sich ihrer Verantwortung für die psychische Gesundheit ihrer Schutzbefohlenen und Arbeitgeber ihrer Pflichten gegenüber ihren Mitarbeitenden bewusst sein und einem zu hohen Leistungsdruck entgegenwirken.

5. In der Gesundheitspolitik sollte Enhancement mit Prävention, also der Verhinderung von Krankheiten, weder verwechselt noch vermischt werden.

6. Vor allem bei Kindern kann pharmakologisches Enhancement die Freiheit, die Persönlichkeitsrechte und die Persönlichkeitsentwicklung einschränken. Eltern, Bildungseinrichtungen und andere Sorgeberechtigte tragen hier eine besondere Verantwortung, sowohl für das einzelne Kind als auch für die zukünftigen Werte und Massstäbe unserer Gesellschaft.

7. Die gegenwärtige Verschreibungspraxis von Psychopharmaka bei Kindern ist zu überprüfen, die Ursachen des höheren Verbrauchs sind zu klären und die Kinder vor übermässigem Gebrauch zu schützen. 


\section{Zitierte Literatur:}

1 Buchanan A, Brock D W, Daniels N, Wikler D. Warum nicht das Beste? In: SchöneSeifert B, Talbot D (eds.). Enhancement: Die ethische Debatte. Paderborn: mentis; 2009. p. 267-295.

2 Harris J. Enhancements are a moral obligation. In: Savulescu J, Bostrom N (eds.). Human Enhancement. Oxford: Oxford University Press; 2009. p. 131-154.

3 Schöne-Seifert B, Talbot D (eds.). Enhancement: Die ethische Debatte. Paderborn: mentis; 2009.

4 Schöne-Seifert B, Ach J S, Opolka U, Talbot D (eds.). Neuro-Enhancement: Ethik vor neuen Herausforderungen. Paderborn: mentis; 2009.

5 Savulescu J, Bostrom N (eds.). Human Enhancement. Oxford: Oxford University Press; 2009.

6 Knoepffler N, Savulescu J (eds.). Der neue Mensch? Enhancement und Genetik. Freiburg/München: Alber Verlag; 2009.

7 Gordijn B, Chadwick R (eds.). Medical Enhancement and Posthumanity. Springer; 2008.

8 Galert T, Bublitz C, Merkel R, Repantis D, Schöne-Seifert B, Talbot, D. Das optimierte Gehirn. Gehirn\&Geist 2009; 11: p. 1-12.

9 Murray T H. Enhancement. In: Steinbock B (ed.). The Oxford Handbook of Bioethics. Oxford: Oxford University Press; 2007. p. 491-515.

10 Allouche S, Missa J-N (eds.). Enhancement: Ethique et philosophie de la médecine d'amélioration. Paris: Libr. Philos. Vrin; 2009.

11 Gesang B. Perfektionierung des Menschen. Berlin: Walter de Gruyter; 2007.

12 Hildt E, Engels E-M (eds). Der implantierte Mensch: Therapie und Enhancement im Gehirn. Freiburg i. Br.: Alber; 2003.

13 Eßmann B, Brittner U, Baltes D. Die biotechnische Selbstgestaltung des Menschen: Neuere Beiträge zur ethischen Debatte über das Enhancement. In: Philosophische Rundschau; 2011; 58: p. 1-21.

14 Sauter, A, Gerlinger, K. Pharmakologische Interventionen zur Leistungssteigerung als gesellschaftliche Herausforderung. TAB-Arbeitsbericht Nr. 143, im Druck; 2011.

15 Biedermeier F. Die Haltung der Deutschschweizer Bevölkerung zum Pharmazeutischen Cognitive Enhancement: Hypothesen basierend auf einer postalischen Befragung in einer Beispielgemeinde. Unveröffentlichte Dissertation Universität Basel; 2010.

16 Eckhardt A, Backmann A, Marti M, Rütsche B, Telser H. Human Enhancement. TA-Swiss 56; 2011.

17 NEK-CNE. Patientenverfügung: Ethische Erwägungen zum neuen Erwachsenenschutzrecht unter besonderer Berücksichtigung der Demenz. Stellungnahme Nr. 17/2011; Bern. 
18 Lenk C. Therapie und Enhancement: Ziele und Grenzen der modernen Medizin. Münster: LIT Verlag; 2002.

19 SuchtMagazin - Fachzeitschrift für Suchtarbeit und Suchtpolitik. Neuro-Enhancer. 2010; 2.

20 Lieb K. Hirndoping: Warum wir nicht alles schlucken sollten. Mannheim: Artemis \& Winkler; 2010; p. 265-295.

21 Repantis D, Schlattmann P, Laisney O, Heuser I. Modafinil and methylphenidate for neuroenhancement in healthy individuals: A systematic review. Pharmacological Research. 2010; doi:10.1016/j.phrs.2010.04.002.

22 Gerbner S, Berlowitz I, Alvarado V, Cassina M. SECO Stressstudie 2010 : Stress bei Schweizer Erwerbstätigen: Zusammenhänge zwischen Arbeitsbedingungen, Personenmerkmalen, Befinden und Gesundheit. 2011. www.seco.admin.ch (Dokumentation/Publikationen/Studien und Berichte).

23 Sprumont $D$, Monbaron S-P. Le développement humain artificiel communément appelé «human enhancement»: Législation actuelle et besoins de réglementation, survol de la problématique. Etude juridique préliminaire, mandatée par la Commission nationale d'éthique pour la médecine humaine (NEK-CNE); 2010.

24 Coenen C, Gammel S, Heil R, Woyke A. Die Debatte über "Human Enhancement“: Historische, philosophische und ethische Aspekte der technologischen Verbesserung des Menschen. Bielefeld: transcript Verlag; 2010.

25 Lengwiler M, Madarasz, J (eds.). Das Präventive Selbst. Eine Kulturgeschichte moderner Gesundheitspolitik. Bielefeld: transcript Verlag; 2010.

26 US President's Council on Bioethics. Beyond Therapy: Biotechnology and the Pursuit of Happiness. Washington D.C.; 2003.

27 Office fédéral de la santé publique. Evolution du nombre de prescriptions de RITALINE $®$ (méthylphénidate) dans le canton de Neuchâtel entre 1996 et 2000. In: Bulletin. 2002; 15: p. 284-289.

28 Kühne R, Rapold R. Der Bezug von Methylphenidat in der Schweiz: Nicht alarmierend Fragen stellen sich dennoch. In: Schweizerische Ärztezeitung. 2011; 92:34. p. 12951299.

29 Bericht und Antrag des Regierungsrates an den Kantonsrat zum Postulat KR-Nr. 296/2005 betreffend Statistik über die Abgabe von Psychopharmaka an Schülerinnen und Schüler der Zürcher Volksschule. 30. März 2010; KR-Nr. 296/2005.

30 Bader M, Pierrehumbert B, Junier L, Halfon O. Die AufmerksamkeitsdefizitHyperaktivitäts-Störung bei Kindern und Jugendlichen: Bericht über eine 2001 in Morges durchgeführte Studie. Lausanne; 2005.

31 Karsch F. Neuro-Enhancement oder Krankheitsbehandlung? Zur Problematik der Entgrenzung von Krankheit und Gesundheit am Beispiel ADHS. In: Viehöver W, Wehling 
P (eds). Entgrenzung der Medizin: Von der Heilkunst zur Verbesserung des Menschen? Bielefeld: transcript Verlag; 2011.

32 Kipke R. Besser werden. Eine ethische Untersuchung zu Selbstformung und NeuroEnhancement. Paderborn: Mentis Verlag; 2011. 